\title{
Consistent Quantitative Trait Loci in Pea for Partial Resistance to Aphanomyces euteiches Isolates from the United States and France
}

\author{
M. L. Pilet-Nayel, F. J. Muehlbauer, R. J. McGee, J. M. Kraft, A. Baranger, and C. J. Coyne
}

First and fifth authors: UMR INRA-AgroCampus Rennes, Amélioration des Plantes et Biotechnologies végétales, BP 35327,35653 Le Rheu Cedex, France; second author: USDA-ARS Grain Legume Genetics and Physiology Research Unit, Washington State University, 303W Johnson Hall, Pullman, WA 99164; third author: Seneca Foods Corporation, Agricultural Research Department, 711 East Main Street, Dayton, WA 99328; fourth author: U.S. Department of Agriculture-Agricultural Research Service (USDA-ARS), 24106 N. Bunn Rd., Prosser, WA 99350 (retired); and sixth author: USDA-ARS Western Regional Plant Introduction Station, Washington State University, 59 Johnson Hall, Pullman, WA 99164.

Accepted for publication 2 June 2005.

\begin{abstract}
Pilet-Nayel, M. L., Muehlbauer, F. J., McGee, R. J., Kraft, J. M., Baranger, A., and Coyne, C. J. 2005. Consistent quantitative trait loci in pea for partial resistance to Aphanomyces euteiches isolates from the United States and France. Phytopathology 95:1287-1293.

Development of pea cultivars resistant to Aphanomyces root rot, the most destructive root disease of pea worldwide, is a major disease management objective. In a previous study of a mapping population of 127 recombinant inbred lines (RILs) derived from the cross 'Puget' (susceptible) $\times$ '90-2079' (partially resistant), we identified seven genomic regions, including a major quantitative trait locus (QTL), Aphl, associated with partial resistance to Aphanomyces root rot in U.S. fields (21). The objective of the present study was to evaluate, in the same mapping population, the specificity versus consistency of Aphanomyces resistance

euteiches originating from the United States and France. The 127 RILs were evaluated in the greenhouse for resistance to pure culture isolates SP7 (United States) and Ae106 (France). Using the genetic map previously described, a total of 10 QTL were identified for resistance in greenhouse conditions to the two isolates. Among these were Aph1, Aph2, and $A p h 3$, previously detected for partial field resistance in the United States. $A p h 1$ and Aph3 were detected with both isolates and Aph2 with only the French isolate. Seven additional QTL were specifically detected with one of the two isolates and were not identified for partial field resistance in the United States. The consistency of the detected resistance QTL over two screening environments and isolates is discussed with regard to pathogen variability, and disease assessment and QTL detection methods. This study suggests the usefulness of three consistent QTL, Aph1, Aph2, and $A p h 3$, for marker-assisted selection.
\end{abstract} QTL under two screening conditions (greenhouse and field, by comparison with the previous study) and with two isolates of Aphanomyces

Common root rot, caused by the soilborne fungus Aphanomyces euteiches Drechs., is one of the most serious diseases on pea (Pisum sativum L.) in many parts of the world including North America, northern Europe, Australia, New Zealand, and Japan (20). Because no efficient cultural or chemical methods of disease control are available, the development of resistant varieties is considered a major objective to manage the disease. Pea germ plasm expressing tolerance or partial levels of resistance have been released in the United States and the quantitative inheritance of the tolerance/resistance has been demonstrated $(14,21,25)$. The molecular dissection of the quantitative partial resistance has recently been investigated in order to better understand the genetic basis of the resistance and support breeding efforts. Weeden et al. $(26,27)$ reported that tolerance to Aphanomyces root rot in one field environment in the United States was associated with a major gene in the pea germ plasm line MN313. In our previous study, we identified seven quantitative trait loci (QTL) associated with partial field resistance over 2 years and two locations in the United States, in the cross Puget (susceptible) $\times$ 90-2079 (partially resistant [11]) (21). One of these QTL, named Aphl, was considered "major" since it was consistently detected over years and locations and explained up to $47 \%$ of the variation in one environment.

Corresponding author: M. L. Pilet-Nayel

E-mail address: Marie-Laure.Pilet@rennes.inra.fr

DOI: 10.1094/PHYTO-95-1287

(C) 2005 The American Phytopathological Society
Additional keywords: Pisum sativum.
To investigate the usefulness of Aphanomyces root rot resistance QTL in marker-assisted selection (MAS), the important question of specificity versus consistency of QTL towards screening methods, environments (field, pure culture), and pathogen variability needs to be studied for two important reasons.

First, assessment of resistance in field screening can be difficult because of climatic and soil variations and interactions with other root invading pathogens. Pure culture screenings allow resistance toward chosen isolates of the pathogen to be assessed independently of environmental variations. Few studies have reported on the correlation between Aphanomyces resistance scorings in field and in controlled conditions in pea. Recently, Moussart et al. (18) found good correlation between a root rot index scored in controlled conditions using a French reference isolate and an aerial index scored in French field conditions. Few studies have investigated the correlation between different scoring methods in same growing environment for assessing Aphanomyces resistance in pea. Pilet-Nayel et al. (21) observed good correlation between different scoring criteria (root rot and above-ground indexes, percent dried weight losses) in field conditions (Pullman, WA, 1998).

Second, existence of genetic variability for pathogen virulence is related to genetic variability of resistance factors in the plant. Race-specific resistance genes have been widely described in plants (7). Isolate-specific resistance QTL have also been reported in several plant species $(1,4,8,13,23,24)$. Although the genetic diversity of pea-infecting $A$. euteiches populations has been demonstrated (15), no reports have been published concerning isolatespecific QTL for resistance to Aphanomyces root rot in pea. Two 
recent studies documenting the diversity of pea-infecting strains of $A$. euteiches have been reported. Using 114 isolates from the central and western United States, Malvick and Percich (15) identified four virulence groups based on pathogenicity assays on five differential pea lines, and one major and two minor groups based on random amplified polymorphic DNA (RAPD) markers. There was no correlation between the pathogenic and genotypic diversity described by these authors. Wicker and Rouxel (29) assessed the pathogenic variability of A. euteiches on pea using 109 isolates (88 from France and 21 from New Zealand, North Europe, and North America) and a differential set of six pea genotypes (28). They identified a predominant virulence group that included a wide range of aggressiveness, especially highlighting partial resistance of the germ plasm lines 552 and PI 180693. They identified another group which was specific to U.S. isolates and avirulent on the pea line MN313. The authors also observed that the French isolates were globally more aggressive than isolates from other geographical origins.

The objective of this study was to assess the specificity and consistency of Aphanomyces resistance QTL toward screening conditions and pathogen variability, in the previously studied cross Puget $\times$ 90-2079 (21). QTL associated with greenhouse seedling resistance to two isolates of A. euteiches from different geographical origins (United States and France) were identified, and the genomic localizations of the detected QTL were compared with those previously associated with field resistance in the United States (21).

\section{MATERIALS AND METHODS}

Plant material and fungal isolates. A total of 127 recombinant inbred lines (RILs), from the cross Puget (susceptible) $\times 90$ 2079 (partially resistant), were used for QTL mapping. Each experiment included the two parents of the RILs, two partially resistant lines PI 180693 and 90-2131 (11), and a susceptible garden pea cultivar ('Dark Skin Perfection') as controls.

Two isolates of A. euteiches were used for greenhouse disease experiments. Isolate SP7 (12), isolated from pea in northern Idaho, was used as a reference strain by J. Kraft. Isolate Ae106 (29) was isolated from soil originating from the Eastern Parisian Basin, France, by baiting on alfalfa seedlings (Medicago sativa L. cv. Europe). Ae106 was chosen because it is less aggressive than most French isolates that cause disease symptoms on 90-2079 (29), and in preliminary assays using five French isolates, it gave the greatest differential reaction between the two parents, Puget and 90-2079 (data not shown). SP7 and Ae106 were characterized by Wicker and Rouxel (29) as belonging to the same predominant virulence group as most of the French isolates tested, based on their differential interactions with a set of six pea genotypes.

Greenhouse disease experiment. The RILs and controls were challenged in the greenhouse with pure cultures of each of the two isolates of A. euteiches. RILs and controls were planted using a randomized complete block design with three inoculated replicates and one uninoculated replicate. Each isolate $\times$ genotype $\times$ replicate combination consisted of 15 plants.

Inoculation and disease assessment method followed the procedure of Kraft et al. (12). Zoospores were used as the primary inoculum source. Zoospore inoculum was prepared as previously described (12) and adjusted to a concentration of 100,000 spores per $\mathrm{ml}$. Seeds were planted in flats of coarse grade perlite. Fiveto seven-day-old seedlings were inoculated with the zoospore suspension by pouring $15 \mathrm{ml}$ of inoculum on the 15 seedlings of each genotype $\times$ replicate combination. After 14 days of incubation under greenhouse conditions $\left(25\right.$ to $30^{\circ} \mathrm{C}$ daytime temperatures, 16-h photoperiod, no humidity control), fresh weights of aboveground vegetative matter and roots of the 15 plants were recorded and subsequently dried at $20 \pm 1{ }^{\circ} \mathrm{C}$ in a forced-air drying room prior to recording dry weights. The same measures were carried out for RILs and controls in the uninoculated replicate. By comparison between inoculated and uninoculated replicates, the following resistance scoring criteria were calculated for each isolate experiment and used in the analysis: the percentages of top wet and dried weight losses per plant (TWL and TDL), and the percentages of root wet and dried weight losses per plant (RWL and RDL).

Genetic map, statistical and QTL analysis. The genetic map used for QTL analysis in this study is described in Pilet-Nayel et al. (21). It is comprised of a total of 324 markers (203 amplified fragment length polymorphisms, 100 RAPDs, 11 simple sequence repeats, 7 sequence tagged sites, 6 isozymes, 2 inter simple sequence repeats, and 2 morphological traits) distributed over 13 linkage groups. Nine linkage groups were assigned to the pea consensus genetic map using markers anchored to other published pea linkage maps.

Statistical analysis of experimental designs were carried out for each resistance scoring criterion using the SAS package (SAS Institute, Cary, NC), as described in Pilet-Nayel et al. (21). The statistical model used for analysis of variance (ANOVA) was $P_{i j}=$ $\mu+G_{i}+R_{j}+e_{i j}$, where $P_{i j}$ is the score of the $i$ th RIL in the $j$ th replicate, $\mu$ is the mean of all the data, $G_{i}$ is the $i$ th RIL effect, $R_{j}$ is the $j$ th replicate effect, and $e_{i j}$ is the residual. The assumption of homogeneity of variances by genotype and replicate was tested using the Bartlett's test. Normality of residual distributions was tested using skewness, kurtosis, and Shapiro-Wilk statistics. RILs adjusted means were estimated from ANOVA and used for QTL analysis.

Pearson correlation coefficients between variables assessed for each isolate, between the two isolates, or between greenhouse and field data (21) were calculated from adjusted means.

Mean-based heritability $\left(h^{2}\right)$ was calculated from ANOVA for each resistance variable using the formula $h^{2}=\sigma_{G}^{2} /\left[\sigma_{G}^{2}+\left(\sigma_{e}^{2} / r\right)\right]$, where $\sigma_{G}^{2}$ is the genetic variance, $\sigma_{e}^{2}$ is the residual variance, and $r$ is the number of replicates per line (10).

QTL mapping was conducted by composite interval mapping using the software QTL-Cartographer version 1.21 for MSWindows (S. Wang, C. Basten, and Z. Zeng, 2001; Department of Statistics, North Carolina State University, Raleigh) as described in Pilet-Nayel et al. (21). A mean logarithm of odds (LOD) threshold of 2.8, calculated using the Zmapqtl module of QTLCartographer, was chosen for all the traits based on permutation tests (6) to declare a putative QTL significant, corresponding to a genome-wide $\alpha$ error risk of $5 \%$.

\section{RESULTS}

Greenhouse Aphanomyces resistance data. ANOVA of each greenhouse assay revealed highly significant genotype and replicates effects for all the resistance variables evaluated for the two isolates $(P \leq 0.004$ and 0.0001 , respectively). Residuals after ANOVA were normally distributed according to the Shapiro-Wilk $(P \geq 0.05)$ and/or skewness and kurtosis statistics. Variances of genotypes and replicates were homogeneous according to Bartlett's test $(P>0.02)$. Heritability estimates for the resistance variables ranged from 0.35 (RDL, Ae106) to 0.87 (TDL, Ae106) depending on the resistance criterion used and the isolate (Table 1). Higher heritability values were observed for traits measured on the top parts of the plants, particularly for isolate Ae106.

Frequency distributions of the estimated adjusted means are represented in Figure 1 for each resistance variable $\times$ isolate data combination. The distributions fit normality according to the $\mathrm{S}$ statistics given by QTL-Cartographer $(\alpha=5 \%)$. Means and ranges were similar between the two isolates for RWL and TDL traits, but differed for TWL and RDL traits with average percentages of losses for TWL higher with isolate Ae106 than with SP7 and inversely for RDL. Compared with the parental values, 
transgressive segregants with increased resistance and susceptibility were observed with the two isolates, showing good evidence of quantitative inheritance.

Pearson correlation coefficients (Table 2) were highly significant between all the traits evaluated for isolate SP7 and between traits measured on the same top or root part of the plants for isolate Ae106. Highest correlation was observed between wet and dried weights scored on same parts of plants (roots/tops) for both isolates. Only one significant correlation was identified between TDL traits measured for the two isolates $(r=0.26, P<0.01)$. Significant but low correlations $(P<0.01)$ were observed between several resistance scores assessed in U.S. field conditions (21) and traits measured in the greenhouse for the U.S. isolate SP7. Only one significant but negative correlation $(r=-0.19, P<$ 0.05 ) was found between U.S. field data and the Ae106 French isolate data.

TABLE 1 . Heritability estimates $\left(h^{2}\right)$ and their confidence interval (in parentheses) for resistance to SP7 and Ae106 Aphanomyces euteiches isolates, evaluated using four disease resistance criteria in the Puget $\times 90-2079$ recombinant inbred lines mapping population

\begin{tabular}{lcc}
\hline & \multicolumn{2}{c}{$h^{2}(90 \% \text { confidence interval })^{\mathrm{a}}$} \\
\cline { 2 - 3 } Resistance criterion & SP7 (USA) & Ae106 (FR) \\
\hline \% Top wet weight losses (TWL) & $0.59(0.46-0.68)$ & $0.71(0.63-0.78)$ \\
\% Root wet weight losses (RWL) & $0.57(0.43-0.67)$ & $0.69(0.60-0.76)$ \\
\% Top dried weight losses (TDL) & $0.69(0.59-0.76)$ & $0.87(0.83-0.90)$ \\
\% Root dried weight losses (RDL) & $0.59(0.47-0.69)$ & $0.35(0.15-0.51)$ \\
\hline
\end{tabular}

a The $90 \%$ confidence interval of $h^{2}$ is calculated according to the method of Knapp et al. (10).

\section{$\%$ wet weight losses}
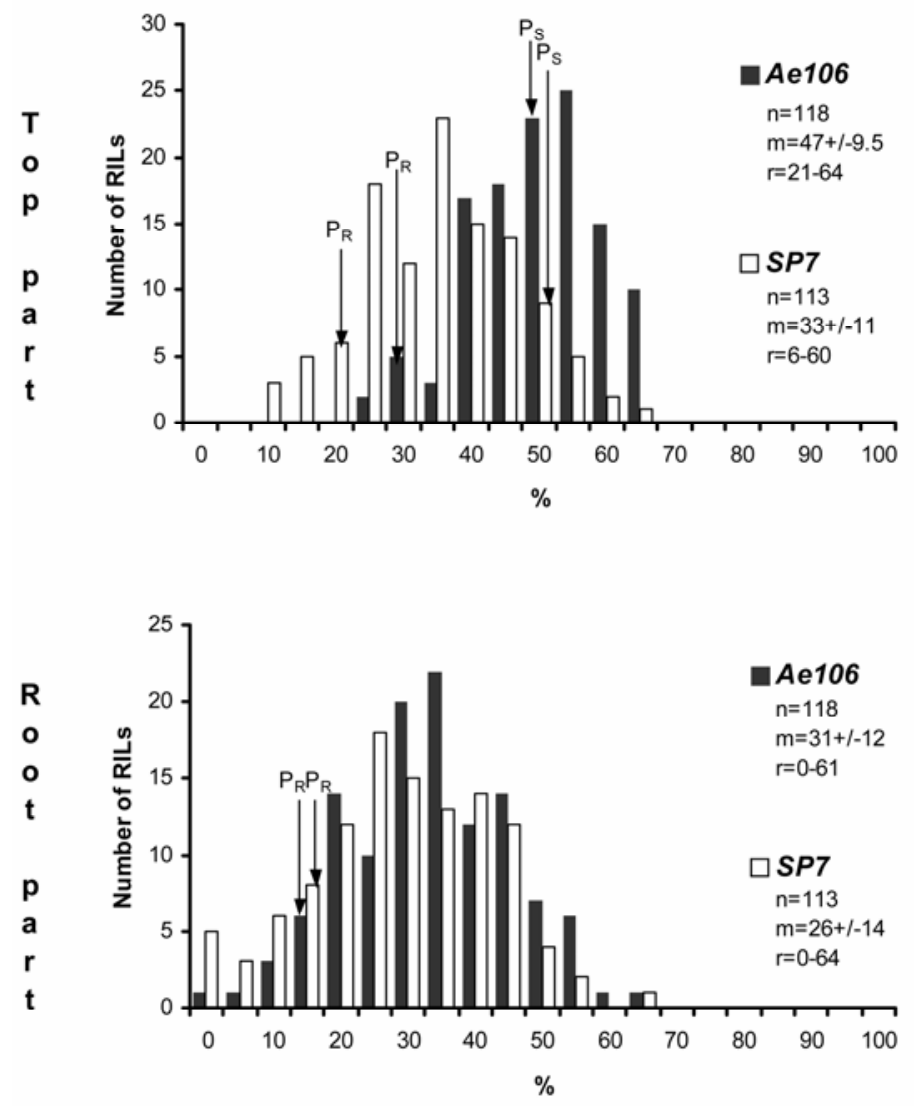

QTL for resistance to $A$. euteiches in greenhouse and comparison with QTL for field resistance. A total of 10 genomic regions, distributed over seven linkage groups, were identified for resistance to SP7 (United States) and Ae106 (French) isolates of A. euteiches in greenhouse-grown seedlings, using four resistance scoring criteria. Statistical detection parameters and genomic localization of these QTL are indicated in Table 3 and Figure 2. Each genomic region explained up to $16 \%$ of the variation, and alleles contributing to resistance came from both the partially resistant (90-2079) and the susceptible (Puget) parent.

Three genomic regions were detected from several traits for resistance to one or both of the isolates. The genomic positions of these three regions coincide with those of the three consistent QTL, Aph1, Aph2, and Aph3, detected for U.S. field Aphanomyces root rot resistance by Pilet-Nayel et al. (21). Accordingly, these three regions were designated as previously named, Aphl, Aph2, and Aph3.

Aphl was identified on linkage group IVb from RWL and TWL traits for isolates SP7 and Ae106, respectively. LOD peaks for the two traits were not detected at identical positions, but they were localized in the confidence interval previously defined for Aphl. The closest markers (U326.190, E7M4.251) of the two QTL peaks coincide with LOD peaks of QTL previously identified for field resistance within the Aphl region. Individually, Aphl explained $11 \%$ of the variation, and the resistance allele at $A p h 1$ was derived from the partially resistant parent, 90-2079. Aphl was considered a major-effect QTL in Pilet-Nayel et al. (21), explaining up to $47 \%$ of the variation in one environment and consistently detected from five resistance variables measured over 2 years and two locations in the United States.

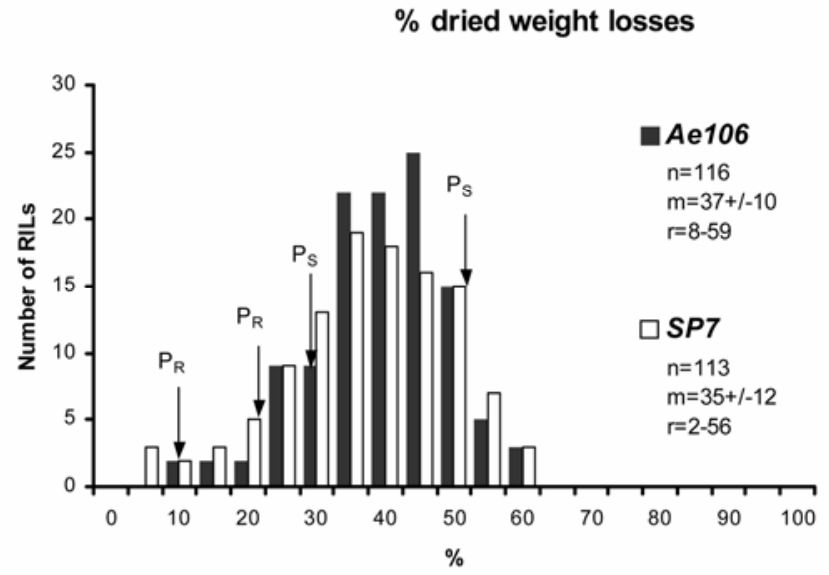

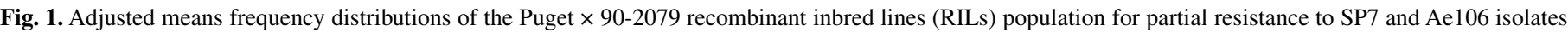

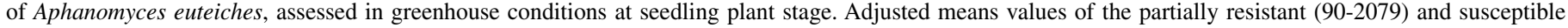

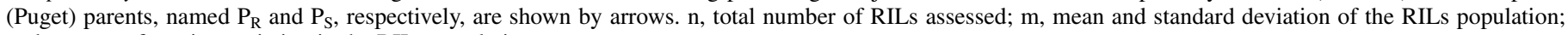
and $r$, range of scoring variation in the RILs population. 
Aph2 was detected on linkage group V from two traits scored on the root parts of the plants for resistance to the French isolate Ae106. It accounted for 11 and $14 \%$ of the variation for RWL and RDL, respectively. Aph2 was identified by Pilet-Nayel et al. (21) from U.S. field scorings in 1996, carried out on roots of plants at Pullman, WA, and on the tops at LeSueur, MN. In both field and greenhouse conditions, the resistance allele at Aph2 was in coupling phase with the $R$ (round seeds) allele. Both alleles are from the partially resistant parent 90-2079.

Aph3 was consistently detected on linkage group Ia for four traits: TWL and TDL with the French isolate Ae106, and TDL and RDL with the U.S. isolate SP7. Aph3 contributed 9 to $16 \%$ of the variation depending on the trait. At Aph3, differences in LOD peak positions and additive effect signs were observed between the RDL trait for isolate SP7 and the three other traits measured on top parts of plants. This suggests that $A p h 3$ would include two distinct genomic regions involved in resistance. In Pilet-Nayel et al. (21), Aph3 was identified for two field resistance scoring criteria assessed in 1998 at Pullman, WA. For the two field traits and one greenhouse trait (RDL with SP7), the resistanceenhancing allele at $A p h 3$ originated from the susceptible parent (Puget), whereas it was derived from the partially resistant parent (90-2079) for the three other greenhouse traits associated with Aph3. The resistance allele derived from 90-2079 is in coupling phase with the af allele that confers afila leaves.

Seven additional genomic regions were significantly detected $(\mathrm{LOD} \geq 2.8$ ) based on data from a single resistance trait for one of the two isolates. None of these regions was detected for field resistance in Pilet-Nayel et al. (21). These QTL were named Aph8 to Aph14 since Aph4 to Aph7 were used in Pilet-Nayel et al. (21) to designate QTL for field resistance. These seven trait-specific QTL individually accounted for 7 to $12 \%$ of the variation.

Three genomic regions, Aph 8 on linkage group III, Aph12 on linkage group VI, and Aphl3 on linkage group VII, were specifically identified for resistance to the French isolate Ae106. Resistance alleles at all three QTL came from the susceptible

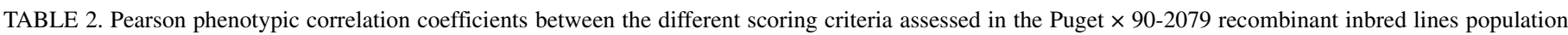

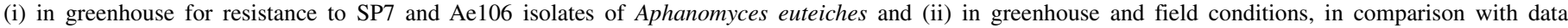
published in Pilet-Nayel et al. (21)

\begin{tabular}{|c|c|c|c|c|c|c|c|c|}
\hline Trait $^{\mathrm{a}}$ & TWL SP7 & RWL SP7 & TDL SP7 & RDL SP7 & TWL Ae106 & RWL Ae106 & TDL Ae106 & RDL Ae106 \\
\hline TWLSP7 & $\ldots$ & $0.45 * * * \mathrm{~b}$ & $0.80 * * *$ & $0.51 * * *$ & $\ldots$ & $\ldots$ & $\ldots$ & $\ldots$ \\
\hline RWLSP7 & $\ldots$ & $\ldots$ & $0.39 * * *$ & $0.82 * * *$ & $\ldots$ & $\ldots$ & $\ldots$ & $\ldots$ \\
\hline TDLSP7 & $\ldots$ & $\ldots$ & $\ldots$ & $0.42 * * *$ & $\ldots$ & $\ldots$ & $\ldots$ & $\ldots$ \\
\hline RDLSP7 & $\ldots$ & $\ldots$ & $\ldots$ & $\ldots$ & $\ldots$ & $\ldots$ & $\ldots$ & $\ldots$ \\
\hline TWLAe106 & 0.01 & -0.02 & 0.02 & -0.06 & $\ldots$ & 0.17 & $0.75 * * *$ & $0.20 *$ \\
\hline RWLAe106 & 0.11 & 0.12 & 0.11 & 0.10 & $\ldots$ & $\ldots$ & 0.04 & $0.68 * * *$ \\
\hline TDLAe106 & 0.16 & 0.00 & $0.26 * *$ & -0.02 & $\ldots$ & $\ldots$ & $\ldots$ & 0.07 \\
\hline RDLAe106 & 0.10 & 0.11 & 0.14 & 0.05 & $\ldots$ & $\ldots$ & $\ldots$ & $\ldots$ \\
\hline AGIMN96 & 0.13 & 0.09 & 0.10 & 0.09 & 0.01 & 0.08 & 0.05 & -0.03 \\
\hline RRIWA96 & -0.09 & 0.02 & -0.11 & 0.05 & -0.15 & 0.12 & $-0.19 *$ & 0.06 \\
\hline AGIMN98 & $0.20^{*}$ & $0.25^{* *}$ & 0.13 & 0.15 & -0.02 & -0.10 & -0.01 & -0.07 \\
\hline AGIWA98 & 0.04 & 0.11 & 0.00 & $0.21 *$ & 0.17 & 0.17 & 0.07 & 0.16 \\
\hline RRIWA98 & -0.01 & 0.15 & -0.10 & $0.19^{*}$ & 0.07 & -0.01 & -0.04 & -0.02 \\
\hline DWLWA98 & 0.17 & 0.11 & 0.11 & 0.15 & 0.15 & 0.02 & 0.14 & 0.01 \\
\hline
\end{tabular}

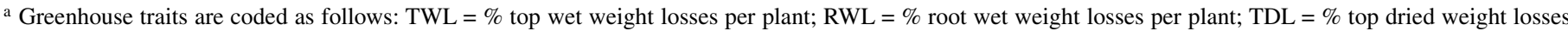
per plant; and RDL $=\%$ root dried weight losses per plant and the name of the isolate used (SP7 or Ae106). Field traits are coded as previously described (21): scoring resistance criterion (AGI, above-ground index; RRI, root rot index; and DWL, \% dried weight losses), location of the scoring (MN: LeSueur, MN; WA: Pullman, WA) and then year of the scoring $(96 ; 98)$.

$\mathrm{b} *, * *$, and $* * *$ indicate significant correlation at $0.05,0.01$, and 0.001 probability levels, respectively.

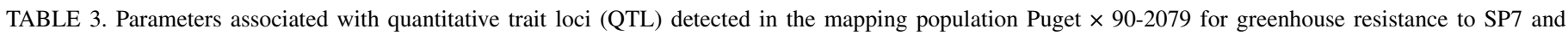
Ae106 isolates of Aphanomyces euteiches using four resistance scoring criteria (values obtained by QTL-Cartographer version 1.21/Windows) (LOD $\geq 2.8$ )

\begin{tabular}{|c|c|c|c|c|c|c|c|}
\hline A. euteiches isolate ${ }^{\mathrm{a}}$ & Scoring criterion $^{\mathrm{b}}$ & QTL name ${ }^{c}$ & Linkage group & Position $(\mathrm{cM})^{\mathrm{d}}$ & Maximum LOD & Additive effect ${ }^{\mathrm{e}}$ & $R^{2}(\%)^{\mathrm{f}}$ \\
\hline \multirow[t]{7}{*}{ SP7 } & \multirow[t]{2}{*}{ TWL } & Aph11 & $\mathrm{IVb}$ & $\mathrm{U} 226.150+0$ & 5.1 & 5.85 & 0.11 \\
\hline & & Aph 9 & Ivb & E6M4.108 + 6 & 3.2 & 3.47 & 0.08 \\
\hline & RWL & Aph1 & $\mathrm{IVb}$ & E7M4.251 + 2 & 3.5 & 4.88 & 0.11 \\
\hline & \multirow[t]{3}{*}{ TDL } & Aph3 & $\mathrm{Ia}$ & $a f+2$ & 5.2 & 5.07 & 0.16 \\
\hline & & Aph10 & $\mathrm{IVb}$ & $\mathrm{U} 530.700+0$ & 3.6 & 4.01 & 0.09 \\
\hline & & Aph14 & A & $\mathrm{E} 3 \mathrm{M} 2.182+2$ & 3.0 & -4.75 & 0.07 \\
\hline & RDL & Aph3 & Ia & $\mathrm{U} 370.900+0$ & 2.8 & -4.30 & 0.09 \\
\hline \multirow[t]{8}{*}{ Ae106 } & \multirow[t]{2}{*}{ TWL } & Aph1 & $\mathrm{IVb}$ & $\mathrm{U} 326.190+0$ & 4.8 & 4.64 & 0.11 \\
\hline & & Aph3 & Ia & E1M3.154 + 2 & 4.6 & 3.45 & 0.12 \\
\hline & \multirow[t]{2}{*}{ RWL } & Aph2 & V & E3M3.167 + 0 & 4.3 & 4.20 & 0.11 \\
\hline & & Aph13 & VII & $\mathrm{E} 8 \mathrm{M} 2.268+4$ & 3.5 & -6.36 & 0.12 \\
\hline & \multirow[t]{2}{*}{ TDL } & Aph3 & $\mathrm{Ia}$ & $a f+2$ & 4.2 & 3.84 & 0.14 \\
\hline & & Aph12 & VI & E1M2.145 + 2 & 3.5 & -3.26 & 0.10 \\
\hline & \multirow[t]{2}{*}{ RDL } & Aph2 & $\mathrm{V}$ & E3M3.167 + 0 & 5.9 & 4.92 & 0.14 \\
\hline & & Aph 8 & III & E7M4.183+ 0 & 2.8 & -3.52 & 0.07 \\
\hline
\end{tabular}

a SP7: A. euteiches isolate from the United States; Ae106: A. euteiches isolate from France.

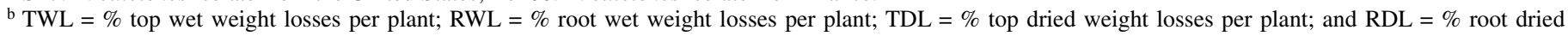
weight losses per plant.

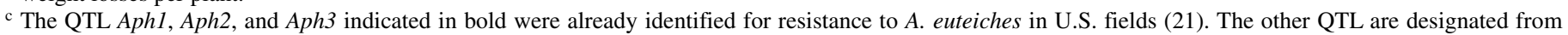
Aph8 to Aph14 since the names Aph4 to Aph7 correspond to other QTL previously identified (21).

${ }^{\mathrm{d}}$ QTL position at the LOD peak from the first marker of the interval (in centimorgans).

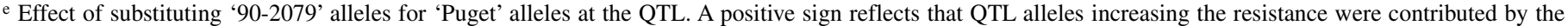
resistant parent '90-2079', whereas a negative sign means that resistance alleles were brought by the susceptible parent 'Puget'.

f Percentage of phenotypic variance explained by an individual QTL. 
LG la

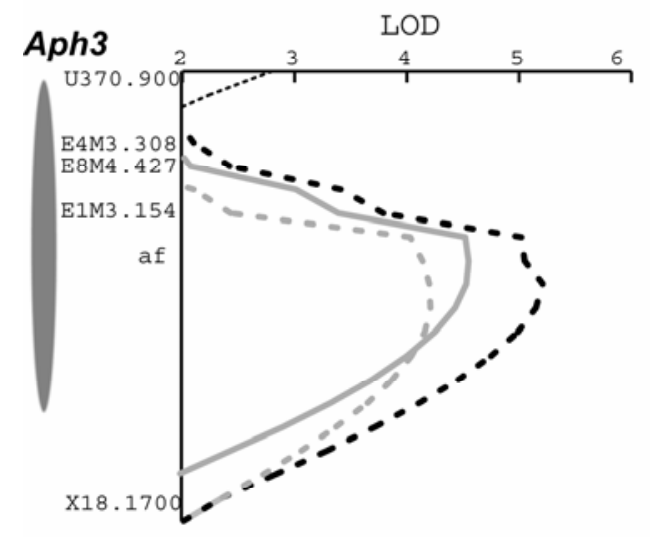

LG V
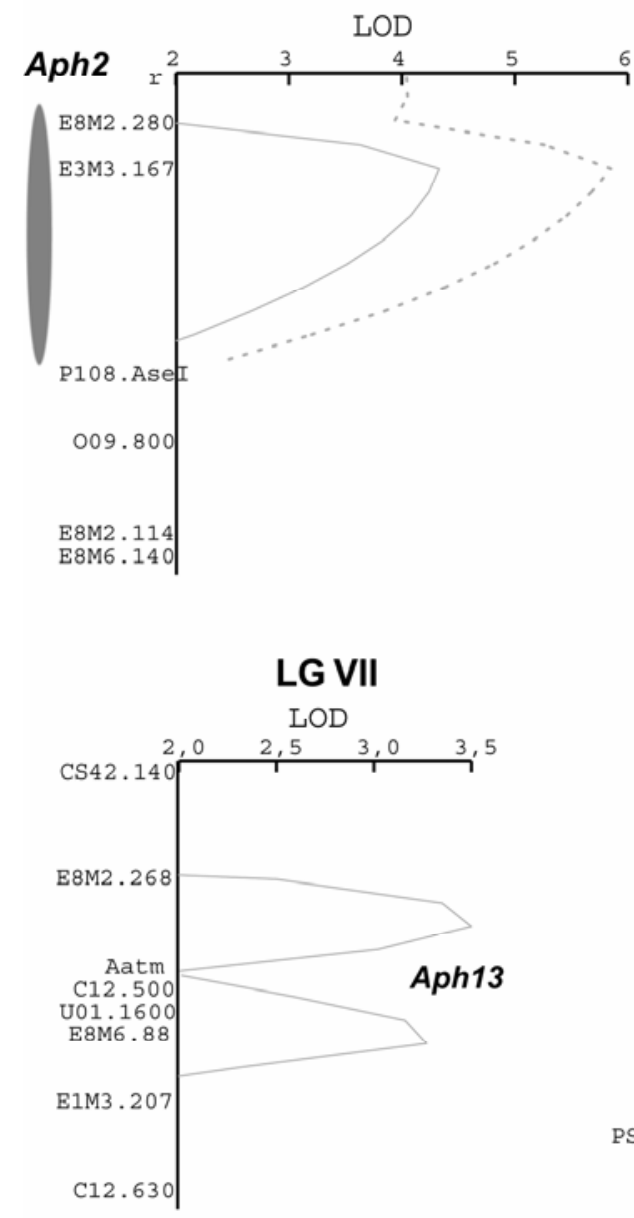

LG III

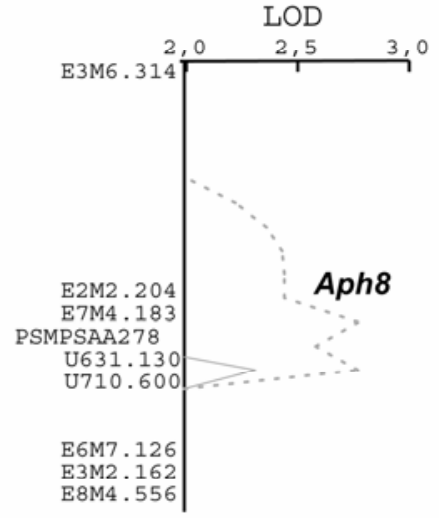

LG VI

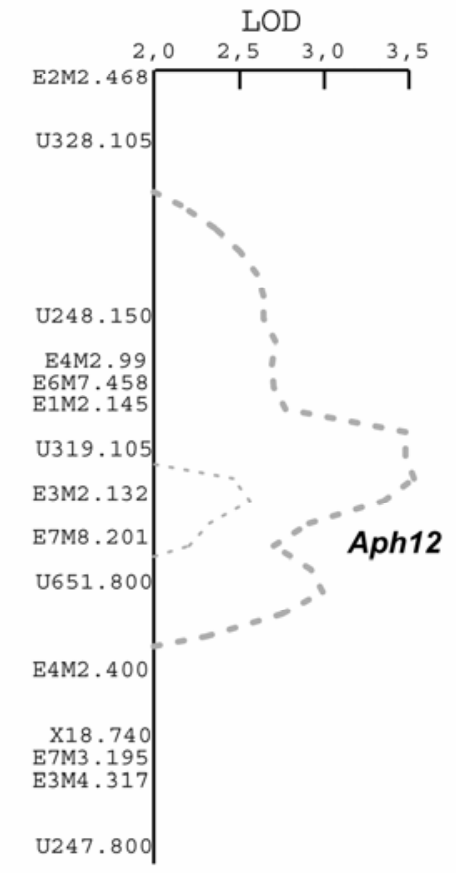

LG IVb

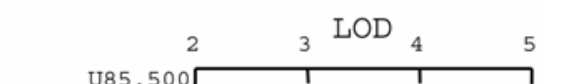

\section{Aph1}

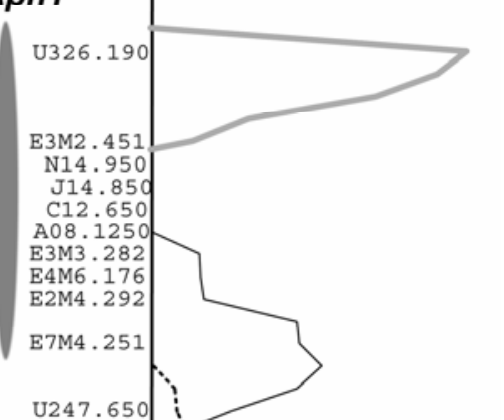

U247.650

E1M8.500

E6M6.135

E8M2.87

E4M6.269

E3M6. 100

E3M2.278

A0 8.2000

A0 8.2000

E1M6. 322

E1M8.472

E3M4. 417

E3M6.141

E7M4.107

E7M2.138

E3M3. 192

E8M3 . 452

E1M4.181.

E8M3. 350

E8M3.99

E4M3.202

E4M3.28

$\mathrm{E} 8 \mathrm{M} 4.640$

U447.100

U79.7 00

$\mathrm{A} 19.400$
$\mathrm{E} 6 \mathrm{M} 4.108$

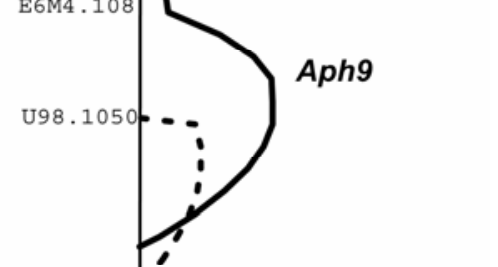

LG A
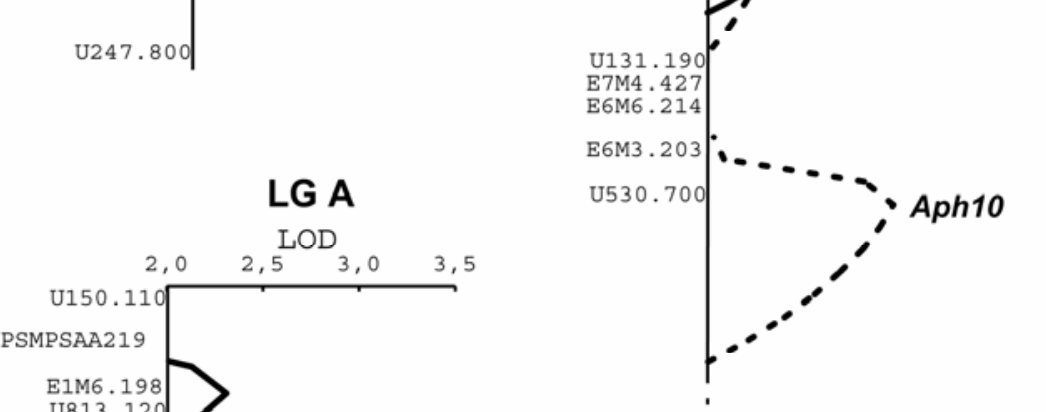

PSMPSAA219

E1M6.198

U813.120

E3M2.182

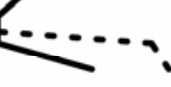

Aph14
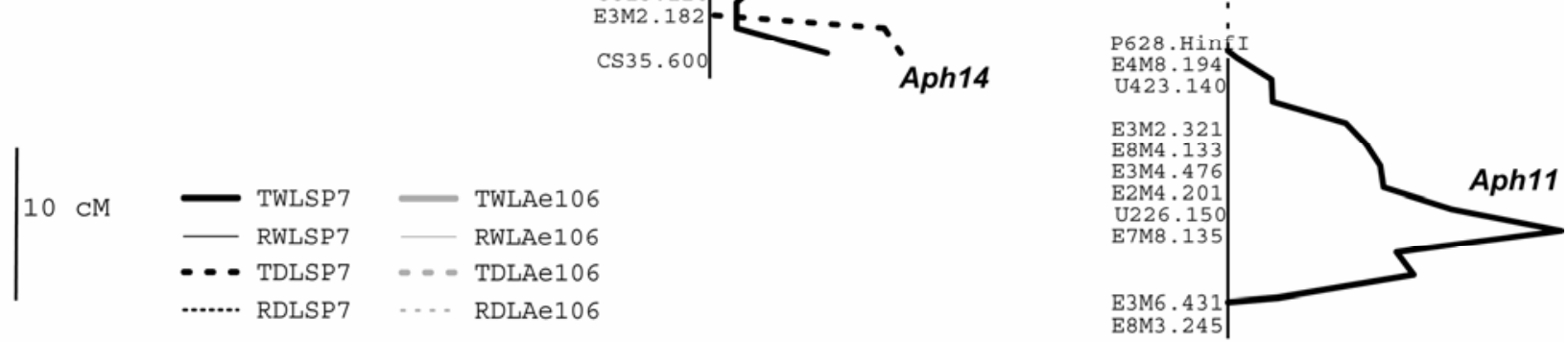

Fig. 2. Logarithm of odds (LOD) curves for quantitative trait loci (QTL) identified in the Puget $\times 90-2079$ recombinant inbred lines (RILs) population for resistance to SP7 and Ae106 isolates of Aphanomyces euteiches in greenhouse. The LOD profiles were obtained using the genetic map previously described (21) and the software QTL-Cartographer version 1.21/Windows (S. Wang, C. Basten, and Z. Zeng, 2001; Department of Statistics, North Carolina State University, Raleigh). Confidence intervals of QTL Aph1, Aph2, and Aph3 previously detected for field resistance (21) are indicated by gray ovals. TWL, percent top wet weight losses per plant; RWL, percent root wet weight losses per plant; TDL, percent top dried weight losses per plant; and RDL, percent root dried weight losses per plant. 
parent. Aph8 and Aph12 were each revealed from one additional trait for resistance to isolate Ae106, with LOD scores ranging from 2.3 to 2.5 , slightly lower than the LOD threshold defined.

Four other regions, Aph9, Aph10, Aph11 on linkage group IVb, and Aph14 on linkage group A, were identified for resistance to the U.S. isolate SP7. Resistance alleles at Aph9, Aph10, and Aph11 were derived from the partially resistant parent. The resistance allele at Aphl4 was derived from the susceptible parent. Aph 9 and Aphl4 were each also detected for one additional resistance trait with the same isolate SP7, at LOD scores ranging from 2.5 to 2.6 .

\section{DISCUSSION}

In this study, we identified a total of 10 QTL associated with greenhouse seedling resistance to two pea-infecting isolates of A. euteiches originating from the United States (SP7) and France (Ae106). Three QTL, Aph1, Aph2, and Aph3, were consistently detected using pure culture screening conditions with at least one of the two isolates studied and field screening conditions. Aphl and Aph3 were consistently detected with both of the isolates studied in pure culture screening conditions. These three QTL correspond to the most consistently detected QTL for field resistance in Pilet-Nayel et al. (21). The seven other QTL, Aph8 to Aph14, were significantly identified specifically from only one of the greenhouse traits used and one of the two isolates. Not all the variation observed for resistance was explained by all the detected QTL. Factors accounting for the unexplained variation presented in Pilet-Nayel et al. (21), including minor effect QTL, incomplete map coverage, epistasis, and environmental effects, are still valid in this study.

Consistency of resistance QTL over screening conditions. In general, low correlation was observed between U.S. field resistance traits and greenhouse resistance traits with the U.S. SP7 isolate. However, Aph1 and Aph3 were each detected from both the U.S. field and SP7 isolate greenhouse evaluations. It has been reported in other crops that same plant resistance QTL have been identified from field and controlled conditions data, even with low or moderately correlated resistance scores between the two different screening conditions $(9,16)$. In our study, there are three possible explanations for the low phenotypic and QTL correlations observed between field and greenhouse conditions.

(i) Differences in environmental (especially soil) conditions, plant developmental stage at scoring and scoring methodologies could have influenced the consistency of field and greenhouse scoring data. Particularly, plants were scored at the flat pod stage in the field, whereas they were scored at the seedling stage in the greenhouse. Different loci may be expressed in the two different development stages. Disease resistance QTL specific to plant developmental stages have been identified in other plants $(5,22,30)$.

(ii) Variability of $A$. euteiches isolates could also explain the differing results observed in this study between field and greenhouse conditions. In the greenhouse, isolates were used in pure culture, whereas in the field, isolates occur in mixture. Additionally, pathogenic characteristics (virulence, aggressiveness) of A. euteiches isolates present in the U.S. field nurseries used (Pullman, WA; and LeSueur, MN) are not known compared with the isolate SP7. Pathogenic variability among U.S. pea-infecting isolates has been reported previously. Malvick and Percich (15) showed that pathogenicity traits differed among 114 populations of A. euteiches from Minnesota, Wisconsin, and Oregon sites, based on their differential reactions on five pea lines. Wicker and Rouxel (29) ranked six U.S. reference isolates, including SP7, in two different virulence groups. SP7 from Idaho was ranked in a predominant virulence group (virulent on six pea lines) and another reference isolate (isolate 467) from Wisconsin in a specific virulence group (avirulent on the pea line MN313). (iii) Occurrence of other root-invading pathogens (Fusarium spp.) in U.S. Aphanomyces field nurseries could also account for the low correlation between U.S. field and greenhouse results in this study. This issue was discussed in Pilet-Nayel et al. (21).

Isolate specificity of resistance QTL? Low correlations were also observed between greenhouse data obtained with the French Ae106 isolate and either U.S. field data or greenhouse data obtained with the U.S. SP7 isolate. Nevertheless, Aph1, Aph2, and Aph3, which were detected from U.S. field and/or SP7 greenhouse data, were also detected in experiments with the French Ae106 isolate. This result is not unexpected as SP7 and Ae106 belong to the same virulence group, according to Wicker and Rouxel (29). This suggests that common genetic factors in pea are involved in resistance to the two isolates. Isolate-specific QTL were also identified for resistance to SP7 and Ae106 isolates. The isolate-specific QTL are considered "minor" since they explain a small part of the variation and were significantly detected based on data for only one trait. Similarly, several studies have reported isolate-nonspecific and isolate-specific QTL in plants that often expressed major and minor effects, respectively, on resistance (1$3,24)$. Interpretation of such results has not been straightforward due to limitations of QTL detection methods. Particularly, minor QTL are difficult to detect because of their small effect on resistance and a relatively large environmental effect. Consequently, minor QTL could be detected based on a single resistance trait for one isolate and could appear specific to one isolate. In our study, four trait-specific minor QTL, Aph8, Aph9, Aph12, Aph14, may be more reliably designated as "isolate-specific" since each of them was also identified from a second trait for the same isolate with the same additive effect sign, although LOD scores were slightly lower than the LOD threshold defined. Moreover, confidence intervals of QTL are often large and not accurately defined, mainly due to the moderate size of the mapping populations studied. As a result, QTL consistently detected from several isolates could correspond to either a single gene carrying alleles involved in different race or isolate specificities or to several clustered genes specific to different isolates or races, as was demonstrated for qualitative resistance (17). Thus, isolate-specific QTL could overlap and be detected as a single region consistently associated with resistance to several isolates. In our study, we suggested that at least two loci in the confidence interval of Aph3 were involved in the resistance to the two isolates studied since differences in positions and parental contributions to the resistance were observed between overlapping QTL detected for each variable in this region.

More precision in QTL detection would be required to understand the genetics of quantitative resistance. It would help to determine whether quantitative partial resistance is due to specific interaction of minor genes from the plant and the pathogen, as suggested by Parlevliet and Zadocks (19), or to the existence of separate gene classes conferring either race-specific or horizontal resistance to different races of pathogens. The use of nearisogenic lines for reliably detecting resistance QTL and of singlespore isolates well-characterized for their virulence genes would contribute to understanding this concept.

This study confirms the usefulness of markers identified in the intervals of three QTL, Aph1, Aph2, and Aph3, for MAS in improving partial resistance to the destructive pea root rotting pathogen A. euteiches.

\section{ACKNOWLEDGMENTS}

This work was funded by post-doctoral fellowships from USDA-ARS (U.S. Department of Agriculture, Agricultural Research Service) and UNIP (Union Nationale Interprofessionnelle des Plantes riches en protéines, Paris, France) and by a grant from INRA-DGAP (Institut National de la Recherche Agronomique, Département de Génétique et Amélioration des Plantes). It was also supported by Washington State 
University, Pullman; USDA-ARS, Prosser, WA; and UMR INRA-AgroCampus Rennes APBV (Amélioration des Plantes et Biotechnologies Végétales), Rennes, France. We thank E. Wicker for providing French isolates; B. Boge, M. McClendon, L. Razai, and L. Johnson for technical assistance for greenhouse RILs phenotyping; and K. Campbell, W. Chen, and T. L. Peever for critical review of the manuscript.

\section{LITERATURE CITED}

1. Arru, L., Francia, E., and Pecchioni, N. 2003. Isolate-specific QTLs of resistance to leaf stripe (Pyrenophora graminea) in the 'Steptoe' $\times$ 'Morex' spring barley cross. Theor. Appl. Genet. 106:668-675.

2. Avila, C. M., Satovic, Z., Sillero, J. C., Rubiales, D., Moreno, M. T., and Torres, A. M. 2004. Isolate and organ-specific QTLs for Ascochyta blight resistance in faba bean (Vicia faba L.). Theor. Appl. Genet. 108:10711078.

3. Calenge, F., Faure, A., Goerre, M., Gebhardt, C., Van de Weg, W. E., Parisi, L., and Durel, C.-E. 2004. A QTL analysis reveals both broadspectrum and isolate-specific QTL for scab resistance in an apple progeny challenged with eight isolates of Venturia inaequalis. Phytopathology 94:370-379.

4. Caranta, C., Lefebvre, V., and Palloix, A. 1997. Polygenic resistance of pepper to potyviruses consists of a combination of isolate-specific and broad-spectrum quantitative trait loci. Mol. Plant-Microbe Interact. 10:872-878.

5. Chantret, N., Mingeot, D., Sourdille, P., Bernard, M., Jacquemin, J. M., and Doussinault, G. 2001. A major QTL for powdery mildew resistance is stable over time and at two development stages in winter wheat. Theor. Appl. Genet. 103:962-971.

6. Churchill, G. A., and Doerge, R. W. 1994. Empirical threshold values for quantitative trait mapping. Genetics 138:963-971.

7. Hammond-Kosack, K. E., and Parker, J. E. 2003. Deciphering plantpathogen communication: Fresh perspectives for molecular resistance breeding. Curr. Opin. Plant Biotechnol. 14:177-193.

8. Huilan, C., Wang, S., Xing, Y., Xu, C., Hayes, P. M., and Zhang, Q. 2003. Comparative analyses of genomic locations and race specificities of loci for quantitative resistance to Pyricularia grisea in rice and barley. Proc. Natl. Acad. Sci. 100:2544-2549.

9. Jung, G., Ariyarathne, H., Coyne, D., and Nienhuis, J. 2003. Mapping QTL for bacterial brown spot resistance under natural infection in field and seedling stem inoculation in growth chamber in common bean. Crop. Sci. 43:350-357.

10. Knapp, S. J., Stroup, W. W., and Ross, W. M. 1985. Exact confidence intervals for heritability on a progeny mean basis. Crop Sci. 25:192-194.

11. Kraft, J. M. 1992. Registration of 90-2079, 90-2131 and 90-2322 pea germplasms. Crop Sci. 32:1076.

12. Kraft, J. M., Haware, M. P., Jiménez-Diaz, R. M., Bayaa, B., and Harrabi, M. 1994. Screening techniques and sources of resistance to root rots and wilts in cool season food legumes. Euphytica 73:27-39.

13. Leonards-Schippers, C., Gieffers, W., Schaüfer-Pregl, R., Ritter, E., and Knapp, S. J. 1994. Quantitative resistance to Phytophthora infestans in potato: A case study of QTL mapping in an allogamous plant species. Genetics 137:67-77.

14. Lewis, M. E., and Gritton, E. T. 1992. Use of one cycle of recurrent selection per year for increasing resistance to Aphanomyces root rot in peas. J. Am. Soc. Hortic. Sci. 117:638-642.
15. Malvick, D. K., and Percich, J. A. 1998. Genotypic and pathogenic diversity among pea-infecting strains of Aphanomyces euteiches from the central and western United Sates. Phytopathology 88:915-921.

16. Mesfin, A., Smith, K., Dill-Macky, R., Evans, C., Waugh, R., Gustus, C., and Muehlbauer, G. 2003. Quantitative trait loci for Fusarium head blight resistance in barley detected in a two-rowed by six-rowed population. Crop. Sci. 43:307-318.

17. Michelmore, R. W., and Meyers, B. C. 1998. Clusters of resistance genes in plants evolve by divergent selection and a birth-and-death process. Genome Res. 8:1113-1130.

18. Moussart, A., Wicker, E., Duparque, M., and Rouxel, F. 2001. Development of an efficient screening test for pea resistance to Aphanomyces euteiches. Pages 272-273 in: Proc. 4th Eur. Conf. Grain Legumes, Cracow, Poland. AEP (ed.), Paris, France.

19. Parlevliet, J. E., and Zadoks, J. C. 1977. The integrated concept of disease resistance: A new view including horizontal and vertical resistance in plants. Euphytica 26:5-21.

20. Pfender, W. 2001. Aphanomyces root rot. Pages 9-13 in: Compendium of Pea Diseases and Pests. 2nd ed. J. M. Kraft and F. L. Pfleger, eds. The American Phytopathological Society, St. Paul, MN

21. Pilet-Nayel, M.-L., Muehlbauer, F. J., McGee, R. J., Kraft, J. M., Baranger, A., and Coyne, C. J. 2002. Quantitative trait loci for partial resistance to Aphanomyces root rot in pea. Theor. Appl. Genet. 106:28-39.

22. Prioul, S., Frankewitz, A., Deniot, G., Morin, G., and Baranger, A. 2004. Mapping of quantitative trait loci for partial resistance to Mycosphaerella pinodes in pea (Pisum sativum L.), at the seedling and adult plant stages. Theor. Appl. Genet. 108:1322-1334.

23. Qi, X., Jiang, G., Chen, W., Niks, R. E., Stam, P., and Lindhout, P. 1999. Isolate-specific QTLs for partial resistance to Puccinia hordei in barley. Theor. Appl. Genet. 99:877-884.

24. Rocherieux, J., Glory, P., Giboulot, A., Boury, S., Barbeyron, G., Thomas, G., and Manzanares-Dauleux, M. J. 2004. Isolate-specific and broadspectrum QTLs are involved in the control of clubroot in Brassica oleracea. Theor. Appl. Genet. 108:1555-1563.

25. Shehata, M. A., Davis, D. W., and Pfleger, F. L. 1983. Breeding for resistance to Aphanomyces euteiches root rot and Rhizoctonia solani stem rot in peas. J. Am. Soc. Hortic. Sci. 108:1080-1085.

26. Weeden, N. F., McGee, R. J., Grau, C. R., and Muehlbauer, F. J. 2000. A gene influencing tolerance to common root rot is located on linkage group IV. Pisum Genet. 32:53-55.

27. Weeden, N. F., McGee, R. J., Hadwiger, L. A., Murphy, R. L., Grau, C. R., Mathre, D. E., Przyborowski, J. A., and Muehlbauer, F. J. 2001. Genetic dissection of tolerance to common root rot Aphanomyces euteiches and other root pathogens in pea. Plant and Animal Genome IX Conf., San Diego, CA. Sherago International Inc., New York.

28. Wicker, E., Moussart, A., Duparque, M., and Rouxel, F. 2003. Further contributions to the development of a differential set of pea cultivars (Pisum sativum) to investigate the virulence of isolates of Aphanomyces euteiches. Eur. J. Plant Pathol. 109:47-60.

29. Wicker, E., and Rouxel, F. 2001. Specific behaviour of French Aphanomyces euteiches Drechs. populations for virulence and aggressiveness on pea, related to isolates from Europe, America and New-Zealand. Eur. J. Plant Pathol. 107:919-929.

30. Williams, K. J., Platz, G. J., Barr, A. R., Cheong, J., Willsmore, K., Cakir, M., and Wallwork, H. 2003. A comparison of the genetics of seedling and adult plant resistance to the spot form of net blotch (Pyrenophora teres $\mathrm{f}$. maculata). Aust. J. Agric. Res. 54:1387-1394. 